\title{
Einleitung: Sterben und Tod öffentlich gestalten
}

Die Thematik „Sterben und Tod“ erfährt in der Gegenwart einen außerordentlichen und anhaltenden Aufschwung: Sterbehilfe ist ein gesellschaftspolitisches Dauerthema; Sterbende machen ihre Erfahrung in Film und Fernsehen, in Büchern und im Internet publik; Studien zum Lebensende haben in der Gesundheitsversorgung enorm an Bedeutung gewonnen, wobei insbesondere die Forschung zu Palliative Care in den Zehnerjahren stark angestiegen ist ${ }^{1}$; die Kulturwissenschaft prägte das wichtige Schlagwort von der „Neuen Sichtbarkeit des Todes“" ${ }^{\text {; }}$ die Soziologie befasst sich ebenfalls seit den Zehnerjahren verstärkt mit Herausforderungen der sozialen Seite des Sterbens ${ }^{3}$, und vermehrt wenden sich der Thematik auch interdisziplinäre Bücher und neu gegründete interdisziplinäre Publikations-Reihen zu. ${ }^{4}$ So ist die Rede von Sterben und Tod heute in den Medien, in den Wissenschaften sowie in den Künsten sehr präsent.

Seit der zweiten Hälfte des 19. Jahrhunderts hatte die moderne säkulare Gesellschaft den Tod „immer entschiedener in den Untergrund verdrängt“. ${ }^{5}$ Gründe hierfür waren die einsetzende Industrialisierung des Bestattungswesens sowie die damit einhergehende Rationalisierung und Institutionalisierung der Kranken- und Sterbeversorgung. Kranke, Sterbende und Tote wurden dem familiären Kontext zunehmend entzogen, ihre Betreuung und Verwaltung ging an Krankenhäuser, Pflegeheime, Hospize und Krematorien über. Mit diesen

1 Vgl. z. B. Heinz Rüegger, Roland Kunz: Über selbstbestimmtes Sterben. Zwischen Freiheit, Verantwortung und Überforderung. Zürich 2020; Markus Zimmermann, Stefan Felder, Ursula Streckeisen, Brigitte Tag: Das Lebensende in der Schweiz. Individuelle und gesellschaftliche Perspektiven. Basel 2019. Siehe auch das nationale Forschungsprogramm NFP 67 des SNF zum Lebensende, 2012-2019: http://www.nfp67.ch/de (letzter Zugriff: 23.11.2021).

2 Thomas Macho, Kristin Marek (Hg.): Die neue Sichtbarkeit des Todes. München 2007.

3 Vgl. beispielsweise das DFG-Projekt Vom ,Guten Sterben' von Christof Breitsameter, Armin Nassehi und Irmhild Saake: https://www.gutessterben.uni-muenchen.de/index.html (letzter Zugriff: 23.11.2021); Thorsten Benkel (Hg.): Die Zukunft des Todes. Heterotopien des Lebensendes. Bielefeld 2016; Martin W. Schnell, Werner Schneider, Harald Joachim Kolbe (Hg.): Sterbewelten. Eine Ethnografie. Wiesbaden 2014.

4 Vgl. z. B. das von Christoph Rehmann-Sutter herausgegebene Buch Was uns der Tod bedeutet (Berlin 2018) oder das neu gegründete Jahrbuch für Tod und Gesellschaft: https://www. hsozkult.de/event/id/event-94921 (letzter Zugriff: 23.11.2021).

5 Philippe Ariès: Geschichte des Todes. München 1980 (frz. OA L'homme devant la mort, 1978), siehe S. $715-815$ (2. Buch, 5. Teil), Zitat S. 718. 
Entwicklungen verschwand das Phänomen des Todes aus dem Alltagsbewusstsein. Die in den 1970er Jahren einsetzende Hospizbewegung sowie die Arbeit der US-amerikanischen Psychiaterin Elisabeth Kübler-Ross gelten heute als „erste Anzeichen einer Enttabuisierung des Themas.“6 Doch eine breitere Diskussion, die in verschiedenen Bereichen gleichzeitig und mit entsprechenden bereichsübergreifenden Referenzen geführt wird, entwickelt sich erst seit den 2o1oer-Jahren. In ihr wird eine "Neue Sichtbarkeit des Todes“ manifest, die heute verschiedene gesellschaftliche Bereiche erfasst hat. Eine wichtige Rolle spielen dabei auch größere Ausstellungen zum Thema, die in den Nullerund Zehnerjahren des 21. Jahrhunderts stattfanden und über die Kunstszene hinaus diskutiert wurden. ${ }^{7}$ Heute ist zudem eine starke Zunahme von Ausstellungen und publikumsnahen Events zu verzeichnen wie beispielsweise das Festival Hallo, Tod! in Zürich (2021), welches auf offene Beteiligung und interaktiven Austausch setzte. ${ }^{8}$ Solche Anlässe tragen zur allgemeinen Sichtbarmachung des Themas bei und fördern durch stark partizipative Ansätze die Weiterentwicklung von entsprechenden gesellschaftlichen Praktiken.

So lässt sich zu Beginn der 2020er-Jahre konstatieren, dass wir uns hinsichtlich der Thematik „Sterben und Tod“ in einer dynamischen experimentellen Periode befinden, die durch stark mediengeprägte Praktiken und Diskurse gekennzeichnet ist, welche ihrerseits in schnellem Wandel begriffen sind. Dabei steht immer auch die Frage auf dem Prüfstein, ob und wie sich die öffentliche Verhandlung von „Sterben und Tod“ erneuern lässt. Exemplarisch hierfür ist die Entwicklung von Sterbeblogs zu nennen. Mit dem Internet hat sich ein neuartiger medialer Darstellungsraum für eine tendenziell niederschwellige Artikulation und Veröffentlichung von Sterbeerfahrungen herausgebildet: In Form von Text- und Videoblogs berichten sterbende Personen heute in real-time vom Verlauf ihrer tödlichen Erkrankungen.

Sowohl die Blogs wie auch manche künstlerische Projekte zu Sterben und Tod führen mitunter zu Skandalisierungen und Kontroversen, die sich an normierten Grenzziehungen zwischen „öffentlich-nicht öffentlich“ bzw. „öffentlich-privat“ entzünden. In den Künsten betrifft dies etwa die Kritik an Fotograf:innen, welche verstorbene Familienangehörige oder unbekannte

6 Zimmermann/Felder/Streckeisen/Tag (2019): Das Lebensende in der Schweiz, S. 11.

7 Z. B. Zum Sterben schön! Der Tod in Literatur, Bildender Kunst und Musik (Goethe-Museum Düsseldorf 2006-2007); Six Feet Under. Autopsie unseres Umgangs mit Toten (Kunsthalle Bern 2007); Das letzte Bild. Fotografie und Tod (C/O Berlin 2018-2019).

8 Vgl. dazu: https://alice-museum-fuer-kinder.fez-berlin.de/ausstellungsverleih/erzaehl-mirwas-vom-tod/; https://www.kunstleben-berlin.de/event/tod-in-der-galerie-verein-berlinerkuenstler/; https://www.hallo-tod.com/ (letzter Zugriff: 23.11.2021). 
Tote fotografieren und die Bilder anschließend veröffentlichen, oder auch die lautstarken Einwände gegen den (nicht realisierten) Vorschlag des bildenden Künstlers Gregor Schneider, sterbenden Menschen im Museum einen realen und damit vom Publikum einsehbaren Sterberaum anzubieten. Solche Kontroversen gründen in differenten Auffassungen darüber, was an die Öffentlichkeit gehört und was nicht. Dabei treten Vorstellungen hervor, wer in der Öffentlichkeit über Sterben und Tod sprechen darf (bzw. wer nicht), und wie dies zu tun ist (bzw. wie nicht).

\section{Sterbeblogs im Internet}

Realtime-Berichte über Krankheits- und Sterbeverläufe im Internet sind seit Mitte der Zehnerjahre exponentiell angestiegen. Einer der ersten nachgewiesenen Berichte stammt von Derek K. Miller (1969-2011), einem kanadischen Blogger der ersten Stunde, der sich u. a. mit Musik und Computerthemen befasste. Im Jahr 2000 postete er seinen ersten Blog, 2005 folgte der erste Podcast. Im Januar 2007 teilte Miller seiner Leserschaft mit, dass bei ihm Darmkrebs diagnostiziert worden sei, und in den kommenden Jahren informierte er Schritt für Schritt über den Krankheitsverlauf bis zu seinem Tod im Mai 2011 (vgl. Abb. 1).

Im deutschsprachigen Raum kann Wolfgang Herrndorfs Blog Arbeit und Struktur (2010-2013) als erster digitaler Sterbebericht gelten, der größere Bekanntheit erlangte und 2013 postum auch in Buchform erschien (vgl. Abb. 2 und 3$)^{9}$

In der ersten Hälfte der Zehnerjahre waren solche Dokumentationen einer tödlich verlaufenden Krankheit in Echtzeit noch ein Novum; heute gehören sie in Form von Text- und Videoblogs in den Social Media zur Tagesordnung. Die quantitative Zunahme ist so frappant, dass mittlerweile spezialisierte Websites für das Teilen von Krankheits- und Sterbegeschichten entwickelt worden sind. ${ }^{10}$ Die Blogs werden mehrheitlich von jüngeren, digital affinen, schwer an Krebs erkrankten Patient:innen erstellt. Es wird angenommen, dass sie tendenziell über ein fest gefügtes soziales Umfeld und über ein stabiles Selbstwertgefühl verfügen ${ }^{11}$, welches über das Bloggen aufrechterhalten werden kann.

9 Wolfgang Herrndorf: Arbeit und Struktur. Berlin 2013.

10 http://www.whatmattersnow.org; https://www.caringbridge.org; https://www.mylifeline. org; http://www.hospicejourney.org (letzter Zugriff: 23.11.2021).

11 Siehe dazu: Arnulf Deppermann: „Multimediale Narration im Angesicht des Todes“. In: Simon Peng-Keller, Andreas Mauz (Hg.): Sterbenarrative. Hermeneutische Erkundungen des Erzählens am und vom Lebensende. Berlin, Boston, MA 2018, S. 137. 
HOME

BLOG ARCHIVES

ABOUT

BUY MY ALBUM

FREE PODSAFE MUSIC ETC.

INSIDE HOME RECORDING

UP GLOSS \& LAPTOPS

NAVARIK

ARTICLES

PHOTOS

FACEBOOK

TWITTER

MARTIN SIKES MEMORIAL

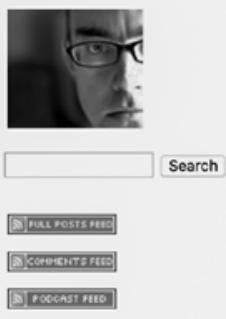

\section{Recently \\ > The last post \\ - I can speak. but in a squeaky way \\ $\rightarrow$ Jean-Hugues \\ > To vote \\ $\rightarrow$ Sometimes \\ > The American junk food situation \\ > On the gravel road \\ $\rightarrow$ So much for being diaper- free \\ - Bring me Diet Cherry Coke and Easy Cheese! \\ The time will come}

\section{Tag Cloud}

\section{family cancer}

geekery death

photography

Vancouver blog chemotherapy.

\section{The last post}

By Derek on May 4, 2011 7:51 AM I 148 Comments I No TrackBacks

Here it is. I'm dead, and this is my last post to my blog. In advance, I asked that once my body finally shut down from the punishments of my cancer, then my family and friends publish this prepared message I wrote-the first part of the process of turning this from an active website to an archive.

If you knew me at all in real life, you probably heard the news already from another source, but however you found out, consider this a confirmation: I was born on June 30, 1969 in Vancouver, Canada, and I died in Burnaby on May 3, 2011, age 41, of complications from stage 4 metastatic colorectal cancer. We all knew this was coming.

That includes my family and friends, and my parents Hilkka and Juergen Karl. My daughters Lauren, age 11, and Marina, who's 13, have known as much as we could tell them since I first found I had cancer. It's become part of their lives, alas.

\section{Airdrie}

Of course it includes my wife Airdrie (née Hislop), Both born in Metro Vancouver, we graduated from different high schools in 1986 and studied Biology at UBC, where we met in '88. At a summer job working as park naturalists that year, I flipped the canoe Air and I were paddling and we had to push it to shore.

We shared some classes, then lost touch. But a few years later, in 1994, I was still working on campus. Airdrie spotted my name and wrote me a letter-yes! paper!-and eventually (I was trying to be a full-time musician, so chaos was about) I wrote her back. From such seeds a garden blooms: it was March ' 94 , and by August ' 95 we were married. I have never had second thoughts, because we have always been good together, through worse and bad and good and great.

However, I didn't think our time together would be so short: 23 years from our first meeting (at Kanaka Creek Regional Park, I'm pretty sure) until I died? Not enough. Not nearly enough.

\section{What was at the end}

I haven't gone to a better place, or a worse one. I haven't gone anyplace, because Derek doesn't exist anymore. As soon as my body stopped functioning, and the neurons in my brain ceased firing, I made a remarkable transformation: from a living organism to a corpse, like a flower or a mouse that didn't make it through a particularly frosty night. The evidence is clear that once I died, it was over.

So I was unafraid of death-of the moment itself-and of what came afterwards, which was (and is) nothing. As I did all along, I remained somewhat afraid of the process of dying, of increasing weakness and fatigue, of pain, of becoming less and less of myself as I got there. I was lucky that my mental faculties were mostly unaffected over the months and years before the end, and there was no sign of cancer in my brain-as far as I or anyone else knew.

As a kid, when I first learned enough subtraction, I figured out how old I would be in the momentous year 2000 . The answer was 31 , which seemed pretty old. Indeed, by the time I was 31 I was married and had two daughters, and I was working as a technical writer and web guy in the computer industry. Pretty grown up, I guess.

Yet there was much more to come. I had yet to start this blog, which recently turned 10 years old. I wasn't yet back playing drums with my band, nor was I a podcaster (since there was no podcasting, nor an iPod for that matter). In techie land, Google was fresh and new, Apple remained "beleaguered," Microsoft was large and in charge, and Facebook and

Abb. 1

Derek K. Miller, Penmachine. 2007-2011. [Blog]. Online unter:

http://www.penmachine.com/ (letzter Zugriff: 23.11.2021). 


\section{ARBEIT UND STRUKTUR

ARCHIV IMPRESSUM

Eins

8.3. $201013: 00$

Gestern haben sie mich eingeliefert. Ich trug ein Pinguinkostüm. Jetzt habe ich einen Panoramablick über ein trapezförmiges Stũck Sprec, den Glaszylinder des Hauptbahnhofs, einen Kanal und klassizistische Gebäude. Auf dem Măuerchen um die Neuropsychiatrie herum sitzt eine Schulklasse. Mein Bedürfnis, unter Zucken und Schreien einen Zettel durchs Fenster hinunterzuwerfen, wächst: „Hilfe! Ich bin nicht verruckt! Ich werde gegen meinen Willen hier festgehalten! Das mit dem Pinguin war nur ein Scherz, ihr konnt Marek fragen oder Verboten Wolf" Aber erstens kann man die Fenster nicht ōffnen und zweitens, fürchte ich. würden sie den Witz nicht kapieren.

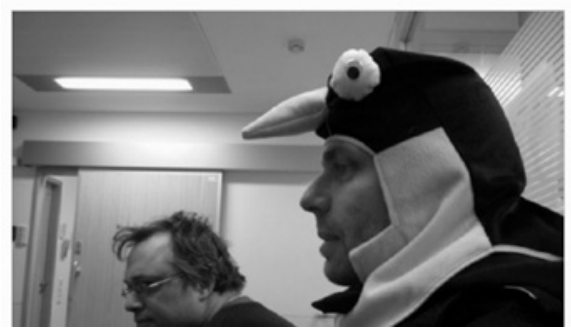

Abb. 2

Wolfgang Herrndorf, Arbeit und Struktur. 2010-2013. [Blog]. Online unter: https://www. wolfgang-herrndorf.de/2010/o4/eins/ (letzter Zugriff: 23.11.2021).

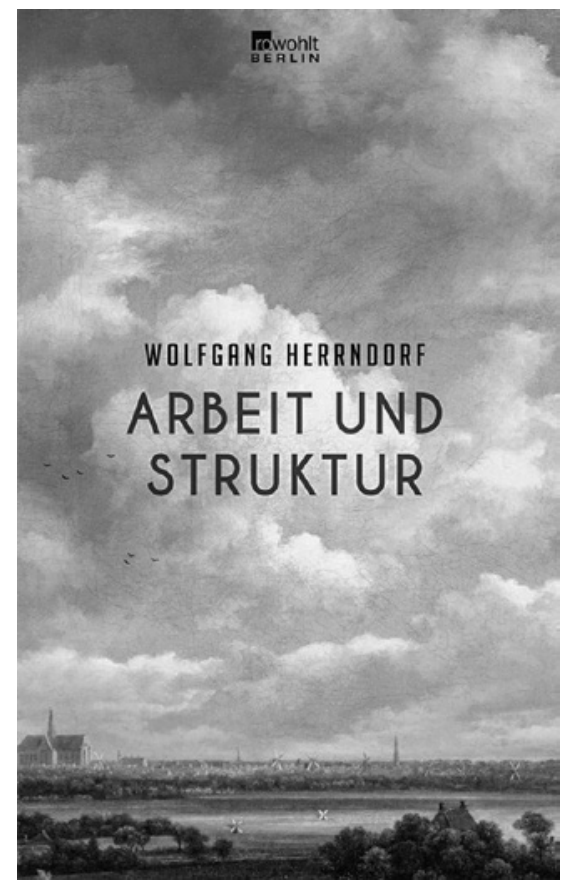

Abb. 3

Wolfgang Herrndorf, Arbeit und Struktur. 2013. [Interneteintrag zur Publikation des rowohlt Verlages]. Online unter: https:// www.rowohlt.de/buch/wolfgang-herrndor f-arbeit-und-struktur-9783499268519 (letzter Zugriff: 23.11.2021).

Nahezu alle Textblogs weisen Archive auf, welche Auskunft darüber geben, wann die Sterbeberichte eingesetzt haben und bis wann sie publiziert wurden. So eröffnete etwa Dmitrij Panov, ein Psychologiestudent aus Marburg, bei dem ein Hirntumor diagnostiziert worden war, seinen Blog Sterben mit Swag im Februar 2016; kurz vor seinem Tod im Oktober desselben Jahres postete er einen letzten Beitrag, mit dem er sich verabschiedete. Andere Blogs umfassen ein, zwei oder auch mehrere Jahre. Oft wird die letzte Nachricht, die den eingetretenen Tod bekannt gibt, von nahen Angehörigen gepostet, wie im Falle von Sylvia Anita Arni-Morf, die von 2014-2016 den Blog Diagnose unheilbar unterhielt und deren Trauerfamilie ihren Tod auf diesem Blog mitteilte. Die meisten Blogger:innen bringen in ihren Texten explizit zum Ausdruck, dass sie im Sterbeprozess begriffen sind - „Yep, I'm dying.“ In vielen Fällen bleiben ihre Blogs auch nach dem Tod im Internet einsehbar (vgl. Abb. 4 bis 6). 


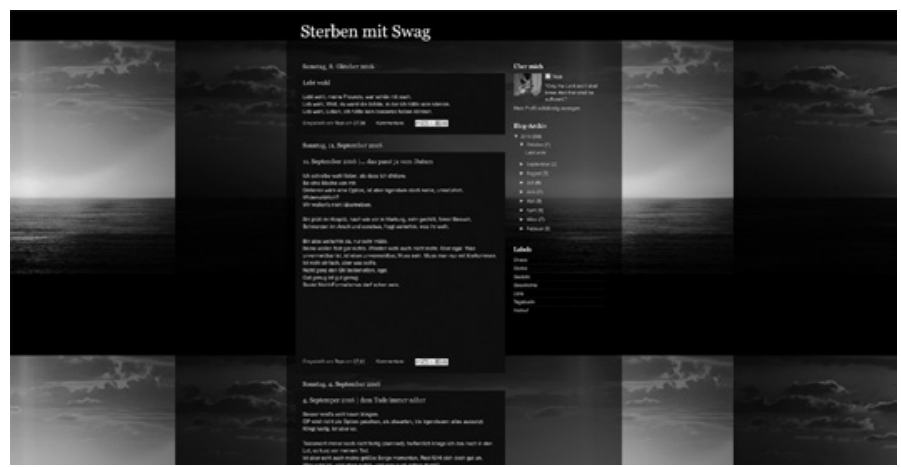

Abb. 4 Dmitrij Panov (DE), Sterben mit Swag. Februar bis Oktober 2016. [Blog]. Online unter: http://sterbenmitswag.blogspot. com/ (letzter Zugriff: 23.11.2021).

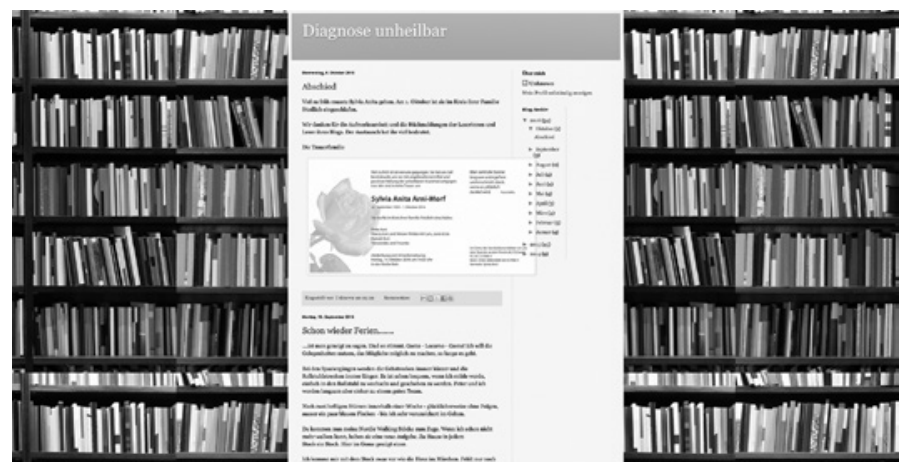

Abb. 5 Silvia Anita Arni (CH), Diagnose unheilbar. Dezember 2014 bis Oktober 2016. [Blog]. Online unter: http://sylviaanita. blogspot.com/ (letzter Zugriff: 23.11.2021).
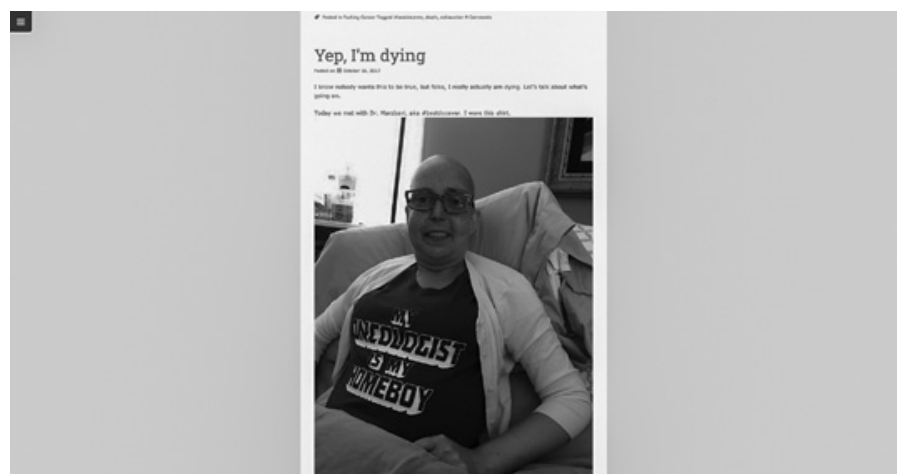

Abb. 6

Beth Caldwell (USA), The Cult of Perfect Motherhood.

Juli 2013 bis November 2017. [Blog]. Online unter: http:// cultofperfectmotherhood.com/ (letzter Zugriff: 23.11.2021). 
In der Regel bestehen die Blogs aus Krankheitsberichten. Geschildert werden die unterschiedlichen medizinischen Therapien, das Auf und Ab der Krankheitsverläufe, Stimmungswechsel, auftretende Schmerzen, Reaktionen des sozialen Umfelds sowie allgemeine Tätigkeiten des (Krankheits-)Alltags. Die Autor:innen können als „wounded storytellers“ bezeichnet werden, wie sie der kanadische Soziologe Arthur W. Frank um die Mitte der 199oer-Jahre in seinem gleichnamigen Klassiker beschrieb: Die narrative Kraft kranker Personen resultiert aus der Wunde. ${ }^{12}$ Jedoch geht es in den neuartigen Sterbeblogs nicht um ein narrativ aufbereitetes Leben mit Verletzung oder Krankheit, sondern um ein Leben mit dem Sterben.

Neben den fortlaufenden Detailschilderungen des Sterbeprozesses findet sich in den Blogs eher wenig Raum für die Beschäftigung mit der verstörenden Ungewissheit über Sterben und Tod und damit verbundenen existenziellen Ängsten. Stimmungsmäßig nehmen sich die Blogtexte oft nicht allzu verzweifelt oder abgründig aus, im Allgemeinen sind sie nicht von radikaler Hinterfragung geprägt. Das mag damit zu tun haben, dass sie sich mehrheitlich an der Alltagssprache und an der Erzählstruktur des Tagebuchs orientieren. - Blogger:innen kommunizieren mit der Öffentlichkeit in Echtzeit, so als würde diese am Bettrand sitzen, zuhören und allenfalls auch reagieren können. Damit sind die Textblogs, obschon monologisch angelegt, an realen dialogischen Kommunikationsverhältnissen ausgerichtet, was eine gewisse Radikalität an lebensabweisenden Formulierungen sowie eine grundlegende Kritik am gesellschaftlichen Umgang mit Sterbenden zu erschweren scheint. Die Motivation zu solchen Blogs mag sich aus unterschiedlichen Bestandteilen zusammensetzen: Ausübung von Selbstbestimmung; Streben nach Anerkennung in Form von Zuspruch und Trost; Selbstreflexion im Sterbeprozess; allgemein starker Kommunikationswunsch; Gestaltung von Hinterlassenschaft; Beitrag zur Enttabuisierung von Sterben und Tod.

Neben dem Textblog stellt auch der Videoblog ein viel beanspruchtes Format für öffentliche Bekundungen von Sterbenden dar. Das Bild- und Tonmedium ermöglicht es auch Personen, die sich nicht gerne schriftlich ausdrücken, sich öffentlich zu äußern. Sie filmen sich mit ihren Handys und bringen dabei ihre Befindlichkeit mündlich zum Ausdruck; anschließend posten sie die Videos. Bei den Videoblogs tritt die Person selbst viel unmittelbarer in den Vordergrund als bei den Textblogs, denn nicht nur die Stimme und das Bewegtbild kommen hier hinzu, sondern auch der kranke Körper selbst rückt in den Fokus: Man blickt direkt auf Krankenbetten, hört entkräftete

12 Arthur W. Frank: The Wounded Storyteller. Body, Illness \& Ethics. 2. Aufl. Chicago, IL, London 2013. 
Stimmen und sieht blutgefüllte Schläuche, elektronische Geräte, am Körper angebrachte Chemotherapie-Beutel, ausgezehrte Leiber, offene Wunden. In solcher Visualität und Tonalität manifestieren sich die Erkrankungen sowie auch die damit einhergehenden mentalen Befindlichkeiten noch unmittelbarer als bei den Textblogs (vgl. Abb. 7 bis 11).

Auch Videoblogs werden chronologisch gepostet, doch kaum archiviert wie die Textblogs, so dass sich ihre Verläufe weniger gut rekonstruieren lassen. Oft finden sich lediglich vereinzelte Postings einer Person. Zudem erfährt man nur in wenigen Fällen, was aus den Videoblogger:innen geworden ist.

Die Blogs manifestieren das heutige Ringen um eine neue Sichtbarmachung und Kommunizierbarkeit der Sterbeerfahrung. Zudem sind sie Ausdruck isolierten Sterbens: Wie Mira Menzfeld in ihrer Studie Anthropology of Dying (2018) aufzeigt, bestehen heute noch kaum Angebote zur Vernetzung von sterbenden Personen, obschon diese sich über ihre Befindlichkeit lieber mit anderen Sterbenden austauschen würden als mit Personen, die sich nicht in der gleichen Situation befinden. Dennoch betreiben sie von sich aus kaum aktive Kontaktsuche, auch nicht qua Internet. ${ }^{13}$ Tatsächlich bieten sich weitgehend monologisch ausgerichtete Sterbeblogs für persönliche Kontaktaufnahmen wenig an, auch wenn sie über Responsefunktionen verfügen. Zweckdienlicher hierfür wären institutionell begründete analoge oder digitale Kontaktforen, die primär für die Vernetzung von sterbenden Personen eingerichtet sind, zwecks Community-Bildung und Austausch auf Augenhöhe.

\section{Öffentlich-nicht öffentlich}

Angesichts der recht unvermittelten Veröffentlichung privaten Leidens rufen die Sterbeblogs Einwände hervor. So bezeichnete etwa der Moderator einer Radiosendung mit dem Titel „Tod ist kein Tabuthema mehr - Kulturwandel beim Reden übers Sterben“ (2020) die Blogs als „unerträglich“; er könne „nicht verstehen, wie Menschen solch intime Vorgänge ins Internet stellen. ${ }^{“ 14}$ Damit wird ein Gegensatz von Öffentlichkeit und Intimität aufgerufen, dem zufolge Sterbeprozesse zu intim, zu privat sind, um öffentlich dargestellt zu werden.

13 Vgl. insb. das Kapitel „Emerging Options for Communitas“. In: Mira Menzfeld: Anthropology of Dying. A Participant Observation with Dying Persons in Germany. Wiesbaden 2018, S. 235-241.

14 Norbert Bischofberger (Mod.), Judith Wipfler (Red.) (2020, 17. November). „Tod ist kein Tabuthema mehr - Kulturwandel beim Reden übers Sterben [Audio-Podcast]“. Mit Corina Caduff und Pascal Mösli. In: SRF2, Kontext. Online unter: https://www.srf. ch/audio/kontext/tod-ist-kein-tabuthema-mehr-kulturwandel-beim-reden-ueberssterben?id=11874348 (letzter Zugriff: 23.11.2021). 
Abb. 7

Ellie Douglas, Chemo Vlog. Day in the Life of a Cancer. 16. Januar 2018. [Videoblog]. Online unter: https://www.youtube. com/watch?v=YO5aooNgSRA, oo:03:24 (letzter Zugriff: 23.11.2021).

Abb. 8

Ellie Douglas, Chemo Vlog. Day in the Life of a Cancer. 16. Januar 2018. [Videoblog]. Online unter: https://www.youtube. com/watch?v=YO5aooNgSRA, oo:o2:55 (letzter Zugriff: 23.11.2021).

Abb. 9

James Kearsley, First Bag of Chemotherapy. Initial Effect. 29. Dezember 2015. [Videoblog]. Online unter: https://www.youtube.com/ watch?v=SxJJWt7jzMc, oo:oo:21 (letzter Zugriff: 23.11.2021).

Abb. 10

James Kearsley, First Bag of Chemotherapy. Initial Effect.

29. Dezember 2015. [Videoblog]. Online unter: https://www.youtube.com/ watch?v=SxJJWt7jzMc, oo:01:23 (letzter Zugriff: 23.11.2O21).
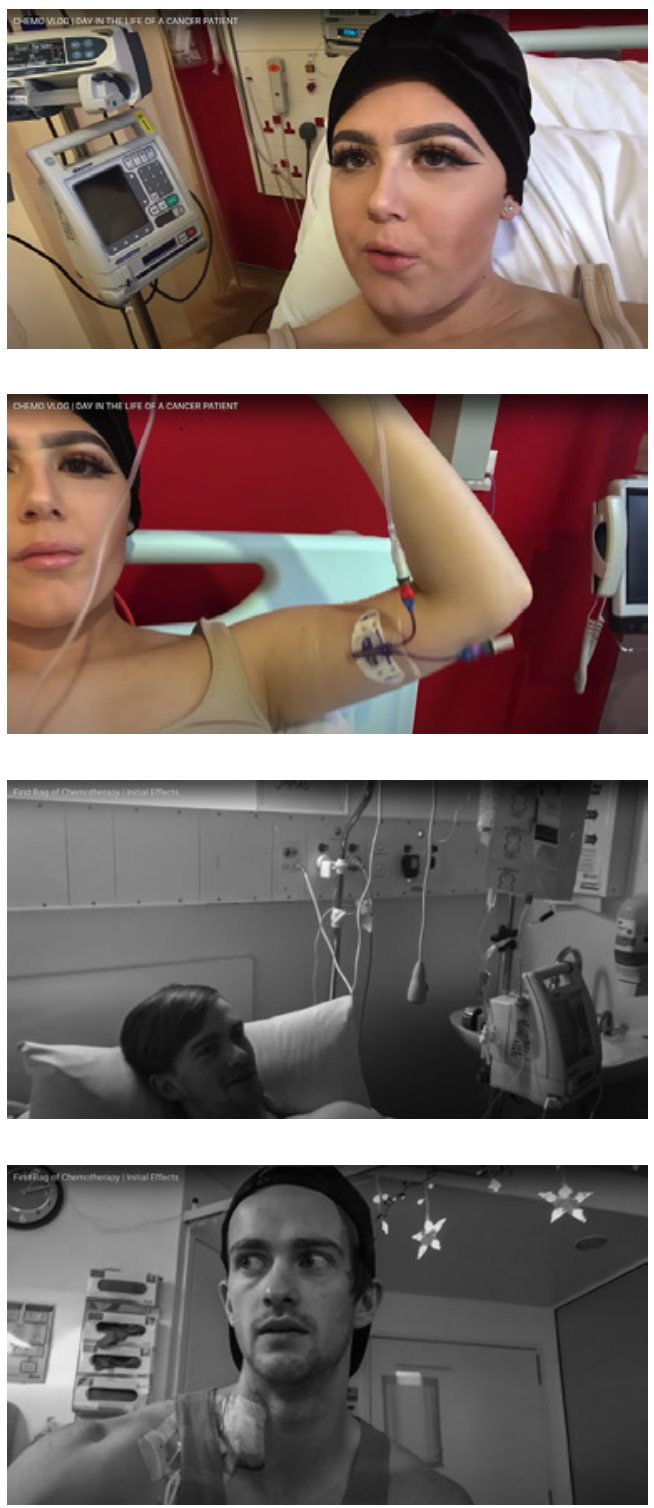

Abb. 11

Vintage Bombshell, My Video Diary through Chemotherapy. og. Februar 2016. [Videoblog]. Online unter: https://www. youtube.com/watch?v=ejnsiRONPRE, oo:o6:26 (letzter Zugriff: 23.11.2021).

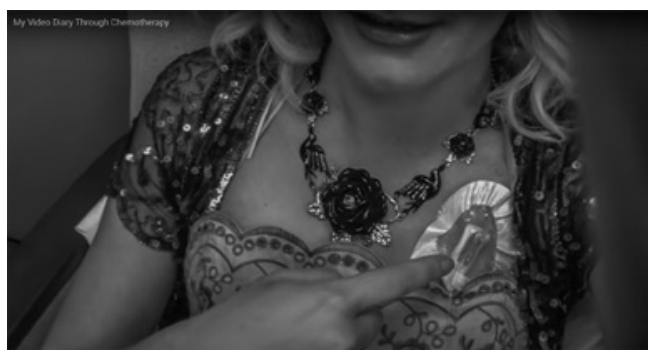


In einer nahezu gleichlautenden Position nahm ein Jahrzehnt zuvor eine Debatte des deutschsprachigen Feuilletons über Sterbebücher ihren Ausgang: „Lasst mich mit eurem Krebs in Ruhe“ - so eröffnete der FAZ-Journalist Richard Kämmerlings 2009 einen polemischen Artikel. Indem er scheinbar fürs Leben votierte, wehrte er die Rede über Tumore, Krebs und Verzweiflung ab: „Lasst uns mit eurem Krebs, eurem Schlaganfall, eurer Leberzirrhose, eurer Schweinegrippe in Ruhe. Erzählt von dem, was zählt, und nicht von Tumormarkern. Erzählt vom Leben. Das Ende kennen wir schon. ${ }^{\text {“15 }}$ Der Journalist Michael Angele doppelte daraufhin im Freitag nach: In einem Artikel mit dem Titel „Wer hat geil Krebs?“, fragte er: „Läge wahre Größe nicht [...] im Verzicht [aufs Sprechen]?"16

Solche Bemühungen, das öffentliche Reden über das Sterben zurückzudrängen, erzeugten damals auch Widerspruch. Allen voran meldete sich der Film- und Theaterregisseur Christoph Schlingensief zu Wort, dessen Buch So schön wie hier kanns im Himmel gar nicht sein. Tagebuch einer Krebserkrankung kurz zuvor erschienen war und im Fokus von Kämmerlings Artikel stand: "Sollen wir alle ganz ehrenhaft schweigen, damit wir diese schreienden Gesundheitsbilder im TV nicht stören? Supermodels, kräftige Haare, weiße Zähne, Adoniskörper [...]?"17 Der Kulturwissenschaftler Thomas Macho betonte im Rahmen der Debatte, dass der Redewunsch von (Tod-)Kranken "nicht in Privaträume und sogenannte Intimzonen verbannt werden darf. ${ }^{418}$ Kämmerlings hatte in seinem Artikel allerdings kein allgemeines Schweigen über den Tod eingefordert, sondern durchaus konkret benannt, was ihn an Krebsbüchern „so unangenehm berührt": „Es ist die Kontamination mit dem Boulevard, der niemand entgeht, der die Tatsachen und Details der Krankheit nicht aussparen kann [...] Man leiht sich dann nämlich, ob man sich dessen bewusst ist oder nicht, die Dramatisierungsformen der Sensationspresse. ${ }^{“ 19}$ Die Krankheit Krebs wird von dem Journalisten dementsprechend als „Boulevardstoff" bezeichnet.

So wird ein sich wiederholendes Argumentationsmuster deutlich: Während auf der einen Seite der Wunsch nach Enttabuisierung, nach Diskussion oder nach allgemeiner Aushandlung an die Öffentlichkeit strebt, wird dieser Wunsch von der anderen Seite her abgedrängt mit dem Verweis darauf,

\footnotetext{
15 Richard Kämmerlings: „Krebsliteratur. Der Schleier über den letzten Dingen“. In: Frankfurter Allgemeine Zeitung, 14. August 2009.

16 Michael Angele: „Wer hat geil Krebs?“. In: Der Freitag, 3. September 2009.

17 Schlingensiefs Replik figurierte als erster von vielen Kommentaren zu Angeles Zeitungsartikel und wurde später in das Buch Ich weiß, ich war's übernommen, S. 22.

18 Thomas Macho: „Wer redet, ist nicht tot“. In: NZZ, 19. November 2009.

19 Kämmerlings (2009): Krebsliteratur.
} 
dass entsprechende Äußerungen nicht öffentlichkeitsrelevant oder nicht öffentlichkeitsverträglich seien. Solche Diskussionen verlaufen entlang der Dichotomien „öffentlich-privat“ bzw. „öffentlich-intim“. ${ }^{20}$ Sie zeugen vom Aufeinandertreffen unterschiedlicher diskursiver Kräfte, welche um eine Neuverhandlung bzw. Beibehaltung entsprechender Grenzen ringen.

In diesem Zusammenhang ist auch die Reality-TV-Show „Over Mijn Lijk“ bemerkenswert, die vom niederländischen Sender BNN seit 2006 zweijährlich im Format einer Dokusoap ausgestrahlt wird. Als Protagonist:innen agieren in jeder Staffel fünf junge Menschen, die an tödlichen Erkrankungen leiden. Sie werden über Monate hinweg von Kamerateams begleitet und dabei im Kreis von Angehörigen und Freund:innen oder bei Gesprächen mit Gesundheitsfachpersonal gefilmt und interviewt. Flankierend zu den Filmaufnahmen bindet der Fernsehsender Onlineblogs der Hauptfiguren sowie weitere Online-Inhalte ein und stellt so gezielt Schnittstellen zwischen Fernsehen und Internet her. Zum Zeitpunkt der Ausstrahlung ist die Mehrheit der porträtierten Protagonist:innen jeweils bereits verstorben. ${ }^{21}$ Als der Sender die preisgekrönte und von anderen Ländern adaptierte Reality-TV-Show ${ }^{22}$ vor eineinhalb Jahrzehnten lancierte, bestand der Tabubruch nicht nur in der öffentlichen Präsentation von tödlich erkrankten jungen Menschen, sondern vor allem auch in der Wahl des Boulevardformats.

„Over Mijn Lijk“ und die Vielzahl gegenwärtiger Sterbeblogs werfen die grundlegende Frage auf, ob die neuen medialen Formate in produktiver Weise zu einer gesellschaftlichen Enttabuisierung von Tod und Sterben beitragen, oder ob sie lediglich eine weitere Form von Selbstbespiegelung qua Social Media oder Dokusoap darstellen, die auch vor dem Tod nicht Halt macht.

Zum Vergleich wäre etwa an die vielen sterbenden Personen zu denken, die sich im Rahmen wissenschaftlicher Forschungsprojekte zu qualitativen Interviews bereit erklären: Zwar werden ihre Daten anonymisiert, aber deren öffentliche Verwendung ist autorisiert, und wohl kaum jemand würde die Interviewbeiträge grundsätzlich infrage stellen. Ähnliches gilt für Dokumentarfilme übers Sterben, die seit den Zehnerjahren eine deutliche Konjunktur erleben, sei es in Form von Einzelporträts oder von filmischen Erzählungen über

\footnotetext{
20 Vgl. dazu auch Reiner Sörries: Öffentliches Sterben. Ein Plädoyer für Intimität. Kevelaer 2014.

21 https://oml.bnnvara.nl/; https://www.instagram.com/overmijnlijk/?hl=de; https://www. facebook.com/overmijnlijk/ (letzter Zugriff: 23.11.2021).

22 Die Sendung erhielt 2006 den Gouden Beelden, 2015 den Zeldzame Engel Award und 2020 den Gouden Televizier-Ring. Das Format wurde von folgenden Sendern übernommen: vom belgischen Sender Eén (2008), vom schwedischen Sender SVT 2 (2008-2015) und vom britischen Sender Channel 4 (2013-2014).
} 
bestimmte Sterbeeinrichtungen ${ }^{23}$ : Auch hier wird den porträtierten Personen nicht vorgeworfen, dass sie sich selbst inszenieren oder dass sie sich filmen lassen. Wenn es dabei gelegentlich zu Einwänden kommt, dann betrifft dies primär die Würde und Distanz der Darstellung bzw. Fragen der Kameraführung und Regie.

Die Vergleiche machen deutlich, dass die Kontroversen um veröffentlichte Sterbeerfahrungen nicht nur die Veröffentlichung per se betreffen, sondern dass sie auch von den jeweiligen Medien, Genres, Formaten und deren Potenzial der Boulevardisierung abhängig sind: Wer darf wie über das eigene Sterben sprechen? Wem und welchem Sprechen mag man zuhören, wem nicht? Damit sind Fragen der Autorschaft und Gestaltung aufgeworfen, die für die Künste eine zentrale Rolle spielen.

\section{Sterben und Tod öffentlich gestalten: Die Beiträge des Bandes}

Gerade anhand von künstlerischen Auseinandersetzungen mit Sterben und Tod lässt sich die Grenzziehung „öffentlich-nicht öffentlich“ um weiterführende Fragestellungen ergänzen: Was für Figurationen von Sterben und Tod werden spezifisch in den Künsten ausgestaltet, welche Sprechweisen, Darstellungsformen und Narrative kommen dabei zum Zug? Wie sind die entsprechenden künstlerischen Arbeiten bezüglich der Grenzziehung zu situieren, wie stellen sie diese in Frage? Was für Wirkungsabsichten verfolgen die Künstler:innen mit ihren entsprechenden Projekten? - Es gehört zum Wesen der Künste, dass sie gesellschaftliche Fragestellungen, Wahrnehmungen und Phänomene künstlerisch ausgestalten, die ansonsten kaum zur Darstellung kommen, und dass sie damit auf die allgemeine öffentliche Diskursbildung einwirken können. So geht es im vorliegenden Band gerade auch um die Frage, inwiefern den Künsten eine Mitwirkung am heutigen gesellschaftlichen Wandel im Umgang mit Sterben und Tod zukommt, inwiefern sie also durch ihre spezifischen

23 Z. B. Ein Sommer für Wenke. Reg. Max Kronawitter. Deutschland 2012; Chi. Reg. Anne Wheeler. Kanada 2013; Joanna. Reg. Aneta Kopacz. Polen 2013; My Love, Don't Cross That River. Reg.Jin Mo-young. Südkorea 2014; Wie wir sterben (3Sat). Reg. Daniela Hoyer, Judith Schneider. Deutschland 2014; Letzte Saison. Wenn es Zeit ist zu sterben (ARD). Reg. Sigrid Faltin. Deutschland 2015; Passing On. Reg. Tom Kleespie. USA 2016; Defining Hope. Reg. Carolyn Jones. USA 2017; Ich sterbe wie ich will (ZDF). Reg. Yves Schurzmann. Deutschland 2017; Mrs. Fang. Reg. Wang Bing. China 2017; End Game (Netflix). Reg. Rob Epstein, Jeffrey Friedman. USA 2018; Island. Reg. Steven Eastwood. USA 2018; Überall wo wir sind. Reg. Veronika Kaserer, Deutschland 2018. 
Darstellungsweisen zu öffentlichkeitsprägenden Ausformungen von neuen Diskursen und Praktiken beitragen, die ohne sie nicht denkbar wären.

Der Band versammelt Einzelstudien zu Beispielen aus Literatur, Kunst und Film aus den letzten zwei bis drei Jahrzehnten. Der zeitliche Fokus liegt dabei hauptsächlich auf Arbeiten, die in den Nuller- und Zehnerjahren veröffentlicht wurden. Neue inhaltliche Schwerpunktbildungen erfolgten dabei insbesondere zwischen 2010 und 2020.

Mehrheitlich basieren die Beispiele auf autobiografischen Erfahrungen der Künstler:innen, das heißt sie betreffen die Erfahrung des eigenen Sterbeprozesses oder die Erfahrung des Sterbens von anderen. Unter beiden Gesichtspunkten lässt sich verfolgen, mit welchen Verfahren die jeweiligen Erfahrungen künstlerisch ausgestaltet werden und wie sich solche Ausgestaltung auf den allgemeingesellschaftlichen Umgang mit Sterben und Tod auswirkt.

Die ersten beiden Beiträge gelten der Literatur. Im Zentrum steht hier ein neues literarisches Genre, das, so die These, aktuell in Entstehung begriffen ist: Autobiografische Sterbeliteratur. Seit den Zehnerjahren ist eine markante Zunahme von literarischen Sterbebüchern zu verzeichnen. Christoph Schlingensief, dessen Tagebuch einer Krebserkrankung von 2009 die erwähnte Feuilleton-Kontroverse mitauslöste, kann als frühes Beispiel gelten. Seither veröffentlichten etliche etablierte Schriftsteller:innen, die schwer erkrankten, letzte Bücher über ihre Sterbeerfahrung, u. a. Jenny Diski, Péter Esterházy und Cory Taylor. Ihnen gemeinsam ist der Wille nach gezielter sprachlicher Gestaltung des Lebensendes, sie beschreiben den körperlichen Zerfall, die voranschreitende gesellschaftliche Ausgliederung, die Erfahrung medizinischer und pflegerischer Betreuung sowie existenzielle Ängste angesichts gravierender Ungewissheiten. In einer vergleichenden Lektüre von mannigfachen, im selben Jahrzehnt verfassten Sterbebüchern eröffnet sich auch der Blick auf gesellschaftliche Muster des heutigen Sterbens (Autobiografische Sterbeliteratur, Kap. 2).

Kontrastierend hierzu folgt ein Beitrag, der sich mit dem Leitmotiv von Sterben, Tod und Toten in den Texten der österreichischen Schriftstellerin Elfriede Jelinek auseinandersetzt. Anders als die autobiografische Sterbeliteratur eröffnet Jelineks Literatur keinen Resonanzraum für eine Besinnung auf das eigene physische Sterben. Vielmehr geht es hier um eine kritische literarische Bearbeitung und Sichtbarmachung von gesellschaftspolitischer Gewalt, welche zum Tod von immer neuen betroffenen Personen und Gruppen führt. Dabei werden insbesondere die strukturellen Aspekte solcher Gewalt offengelegt (Poetiken des Todes bei Elfriede Jelinek, Kap. 3).

Eine weitere Studie befasst sich mit der Darstellung des Leichnams in Film, Literatur, bildender Kunst und Fotografie. Todesverdrängung vollzieht sich 
weitgehend als Totenverdrängung. Die systematische, anhaltende Abdrängung des realen Leichnams aus dem Alltag hat eine visuelle Leerstelle erzeugt, welche insbesondere von Filmen und TV-Serien seit längerer Zeit mit fiktiven Leichnamsbildern besetzt wird; dabei rückt der Leichnam zunächst in der zweiten Hälfte der Nuller- und dann vornehmlich in den Zehnerjahren als Objekt von Bestattungsserien und von forensischen Serien neu ins Zentrum. Auf der anderen Seite setzen sich gegenwärtig insbesondere auch Fotograf:innen, Maler:innen und Performancekünstler:innen mit dem Zeigen von realen Leichnamen auseinander. Die Leistung ihrer Arbeiten besteht darin, den Blick auf diese realen Leichname zu ermöglichen und dabei deutlich zu machen, wie prekär deren Repräsentation in unserer heutigen Kultur ist (Der Leichnam in Film, Literatur und Kunst, Kap. 4).

Eine besondere Herausforderung für die Künste stellen tot geborene Kinder dar. Diese Thematik kommt in verschiedenen Künsten seit einem Jahrzehnt verstärkt zur Darstellung. In Filmen, literarischen Autobiografien und Graphic Novels machen Künstler:innen, welche betroffene Eltern sind, ihre Wahrnehmungen und Befindlichkeiten öffentlich und tragen so zur Weiterentwicklung von bestehenden didaktisch-aufklärerischen Diskursen und Praktiken unterschiedlicher Institutionen bei. In der Diskurs- und Wissensbildung zu tot geborenen Kindern kommt ihnen damit eine besondere Rolle zu (Tot geboren. Babys in Film, Literatur und Internet, Kap. 5).

Ein weiterer Beitrag gilt neueren Jenseitsfilmen. Die Konjunktur solcher Filmerzählungen hält seit den 199oer-Jahren an, doch die narrativen Muster ändern sich. So erfährt etwa das Narrativ der Wiedervereinigung im Jenseits, welches sich seit Beginn der Filmgeschichte kontinuierlich behauptet hat, in den Zehnerjahren einen deutlichen Rückgang, und das ebenfalls seit längerer Zeit beliebte Narrativ Life revisited wird in diesem Jahrzehnt neu akzentuiert. Ebenfalls neu zu verzeichnen ist die Figur des Mediums, die seit ca. 2010 eine allgemeine Karriere im zeitgenössischen Populärfilm macht; dies schlägt sich auch in den Jenseitsfilmen nieder, wo nun Medien an der Schnittstelle zwischen Diesseits und Jenseits als maßgebliche Treiber der Übergänge von der einen Welt in die andere agieren (Jenseitsfilme, Kap. 6).

Insgesamt behandeln die Beiträge sehr unterschiedlich gefasste Sterbe- und Todesreferenzen in den Künsten und machen damit deren aktuelle Spannbreite exemplarisch deutlich. In allen Fällen setzen sich die Veröffentlichungen in Beziehung zu gesamtgesellschaftlichen Diskursen und Praktiken und tragen zu deren Bearbeitung und Veränderung bei. Ein abschließender Beitrag richtet den Blick über die Künste hinaus auf heutige Bestattungspraktiken im Wandel. Er stellt die Treiber dieses Wandels dar - die allgemeingesellschaftliche 
Abkehr von der Kirche und die digitale Transformation - und reflektiert die Veränderungen und das Verhältnis von analogen und virtuellen Friedhöfen (Bestattungspraxis: Figurationen im Wandel, Kap. 7). 